Article

\title{
Development of a Preventive Maintenance Strategy for an Automatic Production Line Based on Group Maintenance Method
}

\author{
Guofa Li, Yi Li, Xinge Zhang *, Chao Hou, Jialong He, Binbin Xu and Jinghao Chen \\ School of Mechanical and Aerospace Engineering, Jilin University, Changchun 130025, China; \\ lgfjlu@163.com (G.L.); liyiccjlu@163.com (Y.L.); houcjlu@163.com (C.H.); hejl@jlu.edu.cn (J.H.); \\ xubinbin@jlu.edu.cn (B.X.); chenjhjlu@163.com (J.C.) \\ * Correspondence: zhangxinge@jlu.edu.cn; Tel.: +86-431-8509-5839
}

Received: 22 August 2018; Accepted: 28 September 2018; Published: 30 September 2018

Featured Application: The preventive maintenance strategy developed in present study can be employed for an automatic production line in the industrial field.

\begin{abstract}
The high maintenance costs and low reliability of automatic production line are attributed to the complexity of maintenance management. In the present study, a preventive maintenance strategy for the automatic production line was developed based on the group maintenance method. The criticality of machines in the production line was evaluated, and then the machines were classified into three groups: the most critical machines, the secondary critical machines and the general machines. The general machines were performed on the breakdown maintenance. The preventive maintenance model of the most critical machines was established with the shortest shutdown time as decision objective on basis of the Delay-time theory. The maintenance model of the secondary critical machine was established based on the considering of reliability-maintenance cost. A case study on an automotive part automatic production line was carried out to verify the proposed preventive maintenance strategy based on the production line data, and the maintenance periods of the most and secondary critical machines were gained; meanwhile, the machines all satisfied the reliability requirements during the maintenance periods.
\end{abstract}

Keywords: automatic production line; preventive maintenance strategy; machine criticality; group maintenance method; maintenance model

\section{Introduction}

An automatic production line is an important part of the manufacturing system in the modern industrial field owing to the enormous advantages of high yield, good product quality and labor cost saving [1-3]. However, due to the variety of machine, complex layout and structure, any machine failure may lead to the whole production line shutdown, resulting in enormous economic losses to the production enterprise. The low reliability and high maintenance costs of the production line have become an urgent problem for production enterprises to solve [4,5]. It is very important to put forward scientific maintenance strategy to guide the maintenance and management works according to the machines' failure characteristics and operation laws of the production line.

Because the working conditions of machines in the production line are different and the machine reliability is also different from each other, if all machines in entire production line are performed on breakdown maintenance or preventive maintenance in the same maintenance period, it is difficult to meet the requirements of high reliability, high operating rate and low maintenance costs [6-8]. In previous literature, the group maintenance method, which meant that a group machines with similar 
characteristics are maintained together in order to reduce the maintenance times and maintenance costs, was considered to be suitable for the automatic production line $[9,10]$. Wu et al. [11] developed an optimized maintenance cost model to determine an optimal interval of condition monitoring and the degradation level after imperfect preventive repairs, which had initiated a new area for the research on cost effective maintenance strategies. Barata and Tong respectively established the maintenance optimization models for the production line using the different methods [12,13]. Rao et al. [14] developed an opportunity maintenance model for the production line to conduct preventive maintenance on several machines at the same time when one of the machines in the production line breaks down. However, it is difficult to use the opportunity maintenance strategy for the complex production line consisting of many machines. Talukder et al. [15] used a heuristic algorithm to group the machines of the production line, and then performed the maintenance to reduce maintenance costs. Arunraj et al. [16] used the Analytic Hierarchy Process (AHP) and goal planning methods to develop the maintenance strategies based on considering the machine failure rate and maintenance costs, respectively. Christer et al. [17] put forward a delay-time model to the prevent machines' failure during the preventative maintenance period when the machines had serious deterioration. However, these previous maintenance models and maintenance strategies were often developed with single evaluation criterion such as reliability and maintenance costs, or the same maintenance model and maintenance strategy were used for all machines in the automatic production line without distinguishing the machines' criticality.

In the present study, a preventive maintenance strategy for the automatic production line was developed based on a group maintenance method. Using the uniform design method, the machines in the production line were classified according to the influence degree of the machine failure rate on the operating rate of the production line. Then, the machines were classified into three groups: the most critical machines, the secondary critical machines and the general machines. The general machines were performed on the breakdown maintenance. The preventive maintenance models were established for the most and secondary critical machines, respectively. The preventive maintenance model of the most critical machines was established with the shortest shutdown time as a decision objective on the basis of delay-time theory, and that of a secondary critical machine was established based on consideration of reliability-maintenance cost. Finally, a case study on an automotive part automatic production line was carried out, and the preventive maintenance periods of the most and secondary critical machines were gained according to the collected data of the production line.

\section{Evaluating of Machine Criticality for an Automatic Production Line}

The operating rate is one of the most important performance indexes to evaluate the automatic production line, which is defined by the ratio of actual output to theoretical output in unit time. The main factors that influence the operating rate of production line are machine failure rate, mean time to repair (MTTR), buffer capacity and production line balancing rate. Because the automatic production line is often a complicated manufacturing system, it is very difficult to establish a definite analytical model between all influence factors and the operating rate. Considering the maintainability, the optimal maintenance strategy directly influenced the failure rate and repair rate of the machines; therefore, the machine failure rate was considered as the key influence factor of the operating rate in this study. The buffer capacity and balancing rate of the production line mostly depended on the design performance of production line and they were taken as the quantitative design parameters into the preventive maintenance model.

For an automatic production line with $K$ machines $\left(M_{1}, M_{2}, \ldots, M_{K}\right)$, the typical layout and structure are shown in Figure 1. 


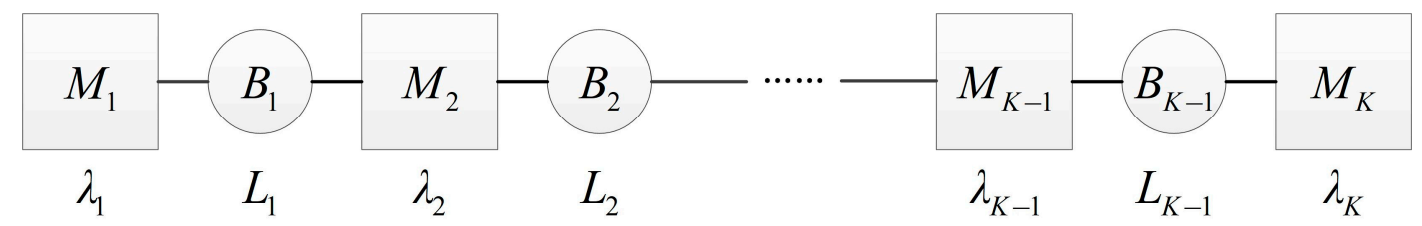

Figure 1. The typical layout and structure of automatic production line, where $B_{i}(i=1,2, \ldots, K)$ is the buffer area, $L_{i}(i=1,2, \ldots, K)$ is the corresponding buffer capacity, $\lambda_{i}(i=1,2, \ldots, K)$ is the machine failure rate, and it is assumed that the time between failure (TBF) of each machine is Weibull distribution.

For the automatic production line, the preventive maintenance is to inspect the machines, and replace parts and lubrication in a certain maintenance period. The preventive maintenance will make the machine return to the state of completely new or good, as long as the parts and lubrication are correctly replaced according to the criteria. The effect of preventive maintenance on the different machine is the same. Therefore, it can be assumed that preventive maintenance has the same effect on the failure rate of each machine, and the operating rates of production line are solved by simulation for the different combinations of the machines' failure rate. In order to ensure the simulation accuracy, many groups of combinations of the failure rate are needed, especially while the production line is comprised of a high number of machines, the simulation calculation will be very difficult and take a long time. To improve the calculation efficiency, the simulation analysis of the production line is carried out in the present study based on the uniform design method [18,19].

First of all, the range of $\lambda_{i}$ value of each machine can be determined according to the machines' failure data. The $N$ levels were taken for each $\lambda_{i}$ ( $N$ is 3-5 times the $K$ of the machines), and then the uniform design table $U_{N}\left(N^{S}\right)$ is established to combine the failure rate for each machine. Finally, the different machines' failure rate combinations are put into the simulation model to calculate the corresponding operating rates of production line $Y_{j}$, and $N$ groups of data samples $\left(Y_{j}, \lambda_{j 1}, \lambda_{j 2}, \ldots\right.$, $\left.\lambda_{j K}\right)(j=1,2, \ldots, K)$ are obtained.

The failure rate of each machine in the production line doesn't affect each other, which means that the variables $\lambda_{j}$ are independent of each other. Therefore, the regression equation of operating rate $Y$ can be expressed as:

$$
\hat{Y}=a+b_{1} \lambda_{1}+b_{2} \lambda_{2}+\cdots+b_{K} \lambda_{K}
$$

where $a, b_{1}, b_{2}, \ldots, b_{K}$ are the unknown parameters in Equation (1).

The fitting regression equation is performed by the minimal mean square error method for the $N$ groups of samples $\left(Y_{j}, \lambda_{j 1}, \lambda_{j 2}, \ldots, \lambda_{j K}\right)(j=1,2, \ldots, N)$ and expressed as:

$$
\min M=E\left(Y_{j}-\hat{Y}_{j}\right)^{2}=E\left(Y_{j}-a-b_{1} \lambda_{j 1}-b_{2} \lambda_{j 2}-\cdots-b_{K} \lambda_{j K}\right)^{2} .
$$

The partial derivation of each parameter in Equation (2) is taken, and the partial derivation is set to be zero. The following equations are gained:

$$
\left\{\begin{array}{l}
E y_{j}=n a+b_{1} E \lambda_{j 1}+b_{2} E \lambda_{j 2}+\cdots+b_{K} E \lambda_{j K} \\
E \lambda_{j 1} y_{j}=a E \lambda_{j 1}+b_{1} E \lambda_{j 1}{ }^{2}+b_{2} E \lambda_{j 1} \lambda_{j 2}+\cdots+b_{K} E \lambda_{j 1} \lambda_{j K} \\
E \lambda_{j 2} y_{j}=a E \lambda_{j 2}+b_{1} E \lambda_{j 1} \lambda_{j 2}+b_{2} E \lambda_{j 2}{ }^{2}+\cdots+b_{K} E \lambda_{j 2} \lambda_{j K} \\
\cdots \cdots \\
E \lambda_{j K} y_{j}=a E \lambda_{j K}+b_{1} E \lambda_{j 1} \lambda_{j K}+b_{2} E \lambda_{j 2} \lambda_{j K}+\cdots+b_{K} E \lambda_{j K}{ }^{2}
\end{array}\right.
$$

where the values of $a, b_{1}, b_{2}, \ldots, b_{K}$ can be gained by solving Equations (3) above. The value of $b_{i}$ indicates the effect of the machine failure rate $\lambda_{i}$ on the operating rate of production line $Y$, and the larger the $b_{i}$, the greater the effect of $\lambda_{i}$ on the operating rate $Y$. 
Finally, the criticality of machine in the production line is evaluated according to the parameter $b_{i}$ in Equation (3), and so the machines can be classified into three groups on the basis of the ABC (Activity Based Classification, ABC) analysis method [20-22]. The principle of ABC classification analysis method comes from Pareto's principle, which classifies the top $15-20 \%$ goods occupied $65-80 \%$ value of whole system into A group, the following 30-40\% goods occupied $15-20 \%$ value of whole system into B group, and the other $40-55 \%$ goods occupied $5-15 \%$ value of the whole system into $\mathrm{C}$ group. For the automatic production line, the most critical machines have an important effect on the operating rate of production line, so the top 20\% (Upper limit of A group ratio in ABC classification method) machines were evaluated as the most critical machines (A group). The secondary critical machines have a lower influence on the operating rate of the automatic production line compared with that of the most critical machines and the production enterprises pay more attention to saving maintenance costs. Thus, the following 30\% (Lower limit of B group ratio in the ABC classification method) of machines were evaluated as the secondary critical machines (B group). The other $50 \%$ of the machines were evaluated as the general machines (C group). Namely, the machines with the parameter $b_{i}$ at the top 20\% are classified into the most critical machines and that the following 20-50\% are classified into the secondary critical machines; thus, the rest of machines are classified into the general machines.

The failure of most and secondary critical machines all have a great influence on the operating rate of production line; therefore, the preventive maintenance should be carried out for them. The failure of general machines has a smaller influence on the operating rate, and the breakdown maintenance is suitable for the general machines.

\section{Preventive Maintenance Modeling for Most Critical Machines Based on Delay-Time Theory}

As mentioned above, the most critical machines as evaluated in Section 2 have great influence on the operating rate of the automatic production line, and they will bring enormous economic losses to production enterprises, if the downtime of the most critical machines is too long. If the preventive maintenance is carried out separately for each of the most critical machines, which causes the frequent shutdown to the production line. Therefore, based on the theory of synchronous maintenance [23], all of the most critical machines are maintained in a group in order to reduce the frequency of the preventive maintenance.

In the present study, the shortest shutdown time was regarded as the decision goal of synchronization maintenance for the most critical machines, and then the balance between the shutdown time of failure and preventive maintenance period was found, so as to minimize the total shutdown time. The relationship between the total expected value of shutdown time $E D\left(T_{m}\right)$ per unit time and the preventive maintenance period $T_{m}$ for the most critical machines can be expressed:

$$
E D\left(T_{m}\right)=\frac{t_{f} E N_{f}\left(T_{m}\right)+t_{p}}{T_{m}}
$$

where $t_{f}$ is the average failure shutdown time, $t_{p}$ is the average preventive maintenance shutdown time, and $E N_{f}\left(T_{m}\right)$ is the expected failure number for all most critical machines in the preventive maintenance period. The $t_{f}$ and $t_{p}$ can be gained by the failure data and maintenance data of the most critical machines. Then, the $T_{m}$ is gained when $E D\left(T_{m}\right)$ is the shortest.

According to the delay-time theory, the machine in the production line may gradually generate the potential failure, which can't cause machine shutdown temporarily. However, if the potential failure is not maintained in time, it will develop into failure and causes the machine to be shutdown. The time while a potential failure develops into a failure is delay-time $t_{h}$. On the basis of the Akaike information criterion, the delay-time $t_{h}$ is exponential distribution [24]. Therefore, the $E N_{f}\left(T_{m}\right)$ of the most critical machines can be calculated according to the delay-time model. The key to establishing the delay-time model is to determine the probability of potential failure $\theta$, delay-time function $f(h)$ and the probability of potential failure that can be detected by preventive maintenance $r$. The maximum-likelihood method 
can be used to calculate the parameters in the delay-time model. Firstly, the preventive maintenance period is divided into $z$ number of $\Delta t$ intervals:

$$
I_{l}^{k}=\left[t_{k-1}+(l-1) \Delta t, t_{k-1}+l \Delta t\right](l=1,2, \cdots, z), t_{k-1}+z \Delta t=t_{k}
$$

where $t_{k-1}$ is the time for the $(k-1)$ th maintenance, and $t_{k-1}+z \Delta t=t_{k}$.

Because the potential failure and its mature failure couldn't generate at the same time, it was assumed that the potential failure and failure were independent events and each potential failure generated independently. Thus, the maximum-likelihood function is expressed:

$$
L=\prod_{k=1}^{n}\left\{\text { Probability of potential failure } n_{k} \text { generating in } T_{k}\right\} \prod_{l=1}^{Z}\left\{\text { Probability of failure } m_{k l} \text { generating in } I_{l}^{k}\right\},
$$

where $n$ is the number of preventive maintenance, $T_{k}$ is the duration time of $k$-th maintenance, $n_{k}$ is the number of potential failures detected in the $k$-th maintenance, and $m_{k l}$ is the number of failures in the $l$-th interval of the $k$-th maintenance period.

While a potential failure of machine generates at time $u$, the probability of the potential failure degrades to be a failure in $(t, t+\Delta t)$ interval is:

$$
p(t, t+\Delta t \mid u)=\left\{\begin{array}{l}
(1-r)^{n-k}(F(t+\Delta t-u)-F(t-u)), T_{k-1}<u<T_{k}, k=1,2, \cdots, n-1 \\
F(t+\Delta t-u)-F(t-u), T_{n-1}<u<t \\
F(t+\Delta t-u), t<u<t+\Delta t \\
0, u>t+\Delta t
\end{array},\right.
$$

where $F\left(t_{h}\right)=1-\exp \left(-\eta t_{h}\right)$ is the distribution function of the delay time $t_{h}$.

While the occurrence of potential failure obeys the Homogeneous Poisson process (HPP) and the occurrence rate is $\theta$, the failure occurrence rate $v(t)$ at time $t$ is expressed:

$$
v(t)=\theta\left(\sum_{k=1}^{n-1}(1-r)^{n-k}\left(F\left(t-T_{k-1}\right)-F\left(t-T_{k}\right)\right)+F\left(t-T_{k-1}\right)\right),\left(T_{n-1}<t \leq T_{n}\right)
$$

From Equation (8), it can be found that the occurrence of failure obeys the Non-homogeneous Poisson Process (NHPP). Thus, the average number of failures during the time interval $I_{l}^{k}$ is:

$$
E N_{f}\left(I_{l}^{k}\right)=\int_{t}^{t+\Delta t} v(t) d t=\theta \int_{t}^{t+\Delta t} \sum_{k=1}^{n-1}(1-r)^{n-k}\left[F\left(x-T_{k-1}\right)-F\left(x-T_{k}\right)\right] d x+\theta \int_{t}^{t+\Delta t} F\left(x-T_{n-1}\right) d x .
$$

The probability of $m_{k l}$ failures generated during the $I_{l}^{k}$ is:

$$
P_{k l}=\frac{\left[E N_{f}\left(I_{l}^{k}\right)\right]^{m_{k l}} e^{-E N_{f}(t, t+\Delta t)}}{m_{k l} !} .
$$

Meanwhile, the probability that the potential failure that generates at time $u$ can be detected in the $T_{k}$ is expressed:

$$
p\left(T_{k} \mid u\right)=\left\{\begin{array}{l}
(1-r)^{k-g} \times r \times\left(1-F\left(T_{i}-u\right)\right), T_{g-1}<u<T_{g}, g=1,2, \cdots, k-1 \\
r \times\left(1-F\left(T_{k}-u\right)\right), T_{k-1}<u<T_{k} \\
0, u>T_{k}
\end{array} .\right.
$$


According to Equation (11), the expected number of potential failures that are detected in the $T_{k}$ can be expressed:

$$
E_{p}\left(T_{k}\right)=\theta \int_{0}^{\infty} p\left(T_{k} \mid u\right) d u=\theta \sum_{l=1}^{k-1}(1-r)^{k-l} r \int_{T_{l-1}}^{T_{l}}\left[1-F\left(T_{k}-u\right)\right] d u+\theta r \int_{T_{k-1}}^{T_{k}}\left[1-F\left(T_{k}-u\right)\right] d u .
$$

As mentioned above, the occurrence of potential failure is assumed to obey the Homogeneous Poisson process (HPP), and, on the basis of Equation (12), the probability of the $n_{k}$-th potential failure detected in the $T_{k}$ is:

$$
P_{k}=\frac{\left(E N_{P}\left(T_{k}\right)\right)^{n_{k}} e^{-E N_{P}\left(T_{k}\right)}}{n_{k} !}
$$

Equations (10) and (13) are taken into Equation (6) and take its natural logarithm, and then the logarithm Likelihood function is gained as follows:

$$
\ln L=\sum_{k=1}^{n}\left[n_{k} \ln E N_{p}\left(T_{k}\right)-E N_{p}\left(T_{k}\right)\right]+\sum_{k=1}^{n} \sum_{l=1}^{z}\left[m_{k l} \ln E N_{f}\left(I_{l}^{k}\right)\right]-\sum_{k=1}^{n} \sum_{l=1}^{z}\left[E N_{f}\left(I_{l}^{k}\right)\right]
$$

The $m_{k l}$ and $n_{k}$ obtained based on the failure and maintenance data of the production line are taken into Equation (14), and the value of $\theta, f\left(t_{h}\right)$ and $r$ can be gained by solving the maximum value of $\ln L$. The $E N_{f}\left(T_{m}\right)$ is gained accordingly to take the $\theta, f\left(t_{h}\right)$ and $r$ into Equation (9). Finally, the preventive maintenance period of the most critical machines can be determined by Equation (4).

According to the above model, the optimal preventive maintenance period for the most critical machines can be calculated. Meanwhile, it is necessary to ensure the reliability of the most critical machine in the preventive maintenance period that can bring the shortest shutdown time. The time between failure (TBF) of the production line is subject to Weibull distribution [17,23]:

$$
F_{w}(t)=1-\exp \left[-(t / \alpha)^{\beta}\right], t \geq 0 .
$$

The maximum likelihood estimation method can be used to solve the scale parameter $\alpha$ and shape parameter $\beta$ of each machine. The cumulative failure probability of each machine can be calculated by taking the preventive maintenance period $T_{m}$ into Equation (15), and then the cumulative failure probabilities are compared with experience value from the literature or specified value from enterprise to confirm.

\section{Preventive Maintenance Modeling for Secondary Critical Machines Based on Reliability-Maintenance Cost}

The secondary critical machines as evaluated in Section 2 have lower influence on the operating rate of the automatic production line compared with that of the most critical machines, and the production enterprises pay more attention to save maintenance costs for the secondary critical machines. Because the failures of the secondary critical machines may also lead to the shutdown of the automatic production line, it is also necessary to ensure the reliability of the secondary machines to ensure the operating rate of production line.

Firstly, the reliability model of the secondary critical machines is established to ensure the machine reliability requirement in the preventive maintenance period. In the reliability model, the failure probability of secondary critical machine needs to be lower than an upper limit. For the $e$ secondary critical machines evaluated in Section 2, the cumulative failure probability $F_{m}\left(T_{s}\right)$ of the $m$-th secondary critical machine during the preventive maintenance period $T_{s}$ for the secondary critical machines is:

$$
F_{m}\left(T_{s}\right)=1-\exp \left[-\left(T_{s} / \alpha_{m}\right)^{\beta_{m}}\right]
$$

where $\alpha_{m}$ and $\beta_{m}$ are the scale parameter and shape parameter in the Weibull distribution, respectively. 
To ensure that $F_{m}\left(T_{s}\right)$ is less than the upper limit $F_{\max }, T_{s}$ must meet the following condition:

$$
T_{s}=\min \left\{T_{s e}\right\}=\min \left\{\alpha_{m}\left[\ln \left(\frac{1}{1-F_{\max }}\right)\right]^{1 / \beta_{m}}\right\},
$$

where $T_{s e}$ is the maximum preventive maintenance period for each secondary critical machine under the reliability constraints.

Then, the maintenance cost model is established to optimize the total maintenance costs of the secondary critical machines during a production period. It is assumed that the TBF of the machine is Weibull distribution. In the preventive maintenance period of the secondary critical machines, the expected failure number of the $m$-th machine is $[25,26]$ :

$$
\operatorname{EN}_{f}^{m}\left(T_{s}\right)=\left(\frac{T_{s}}{\left(\alpha_{m}\right)}\right)^{\beta_{m}}
$$

The total maintenance costs of machine in a production period $T_{J}$ are comprised of the preventive maintenance costs and the machine failure loss, which can be expressed as [27]:

$$
T C\left(T_{s}\right)=n C_{o}+\sum_{m=1}^{e} n_{m} C_{m}+\sum_{m=1}^{e} n_{m} C_{f m}\left(\frac{T_{s e}}{\alpha_{m}}\right)^{\beta_{m}},
$$

where $n$ is the number of preventive maintenance in the production period, $C_{0}$ is the fixed cost for each preventive maintenance, $n_{m}$ is the number of preventive maintenance for the $m$-th secondary critical machine, $C_{m}$ is the cost of parts replacement for the preventive maintenance of the $m$-th secondary critical machine, $C_{f m}$ is the average cost of the breakdown maintenance for the $m$-th secondary critical machine.

In order to minimize the maintenance costs based on meeting the requirement of machine reliability, the multi-objective function of reliability-maintenance cost is proposed:

$$
\begin{aligned}
& \max T_{s} ; \\
& \min T C\left(T_{s}\right)=n C_{0}+\sum_{m=1}^{e} N_{m} C_{m}+\sum_{m=1}^{e} N_{m} C_{f m}\left(\frac{T_{P M m}}{\alpha_{m}}\right)^{\beta_{m}} ; \\
& \quad T_{s e} \geq T_{s} \times Y_{m}, F_{m}\left(T_{s}\right) \leq F_{\max } \\
& \quad T_{s e} \leq \alpha_{m}\left\{\ln \left[\frac{1}{1-F_{\max }}\right]\right\}^{1 / \beta_{m}}
\end{aligned}
$$

where $Y_{m}=\left[T_{s e} / T_{s}\right]$, namely $Y_{m}$ is the integer value of $T_{s e} / T_{s} . T_{P M m}$ is the preventive maintenance period of the $m$-th secondary critical machine and $T_{P M m}=T_{S} \times Y_{m}, N_{m}$ is the number of preventive maintenance for the $m$-th machine in the production period and $N_{m}=\left[T_{J} / T_{P M m}\right], n$ is the number of preventive maintenance in the production cycle and $n=\max \left\{N_{m}\right\}$.

To solve the preventive maintenance model of the secondary critical machines based on the reliability-maintenance cost, the values of the parameters $\left(C_{0}, C_{m}, C_{f m}, F_{\max }, \alpha_{m}, \beta_{m}\right)$ can be gained from the failure and maintenance data of the machines, and they are taken into Equation (16). The preventive maintenance period $T_{P M m}$ of each secondary machine is obtained. $T_{P M m}$ is an integral multiple of $T_{S}$ and the closer it is to $T_{s e}$, the better $T_{P M m}$ is. Meanwhile, the secondary critical machines are grouped for the preventive maintenance according to their $T_{P M m}$, and there are as many machines as possible in each group.

\section{Case Study}

The preventive maintenance strategy developed in present study was used to analyze an automotive part production line located in Shandong Province, China. This production line is comprised of 20 processing machines and it operates $16 \mathrm{~h}$ every day. The machines and layout 
of the automatic production line are shown in Figure 2, and the scene of the production line is shown in Figure 3. The failure rate of each machine was gained accordingly to collect and statistically analyze the production line data including the operating data, failure data and maintenance data. The failure rates of machines in the production line are shown in Table 1.

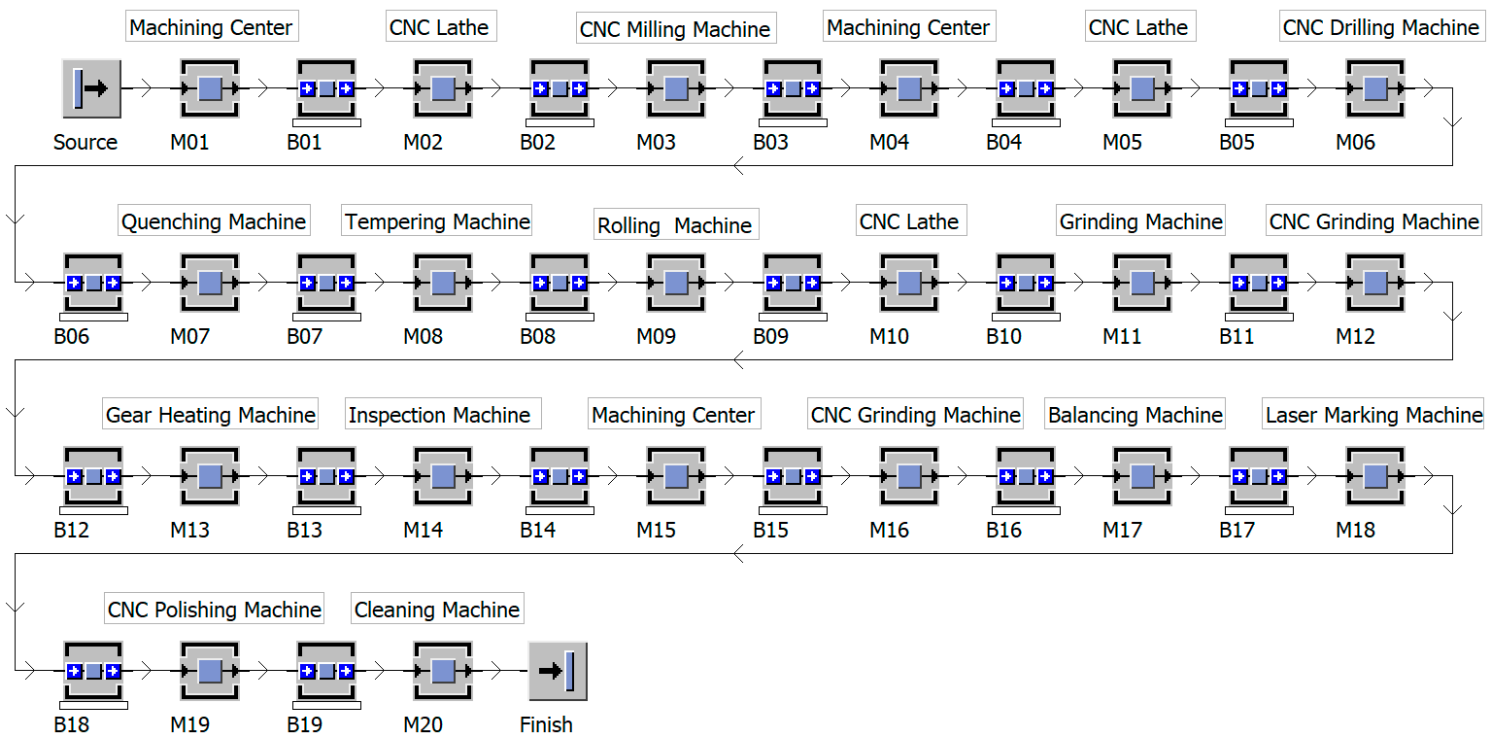

Figure 2. The machines and layout of an automotive part production line (CNC: Computer Numerical Control).

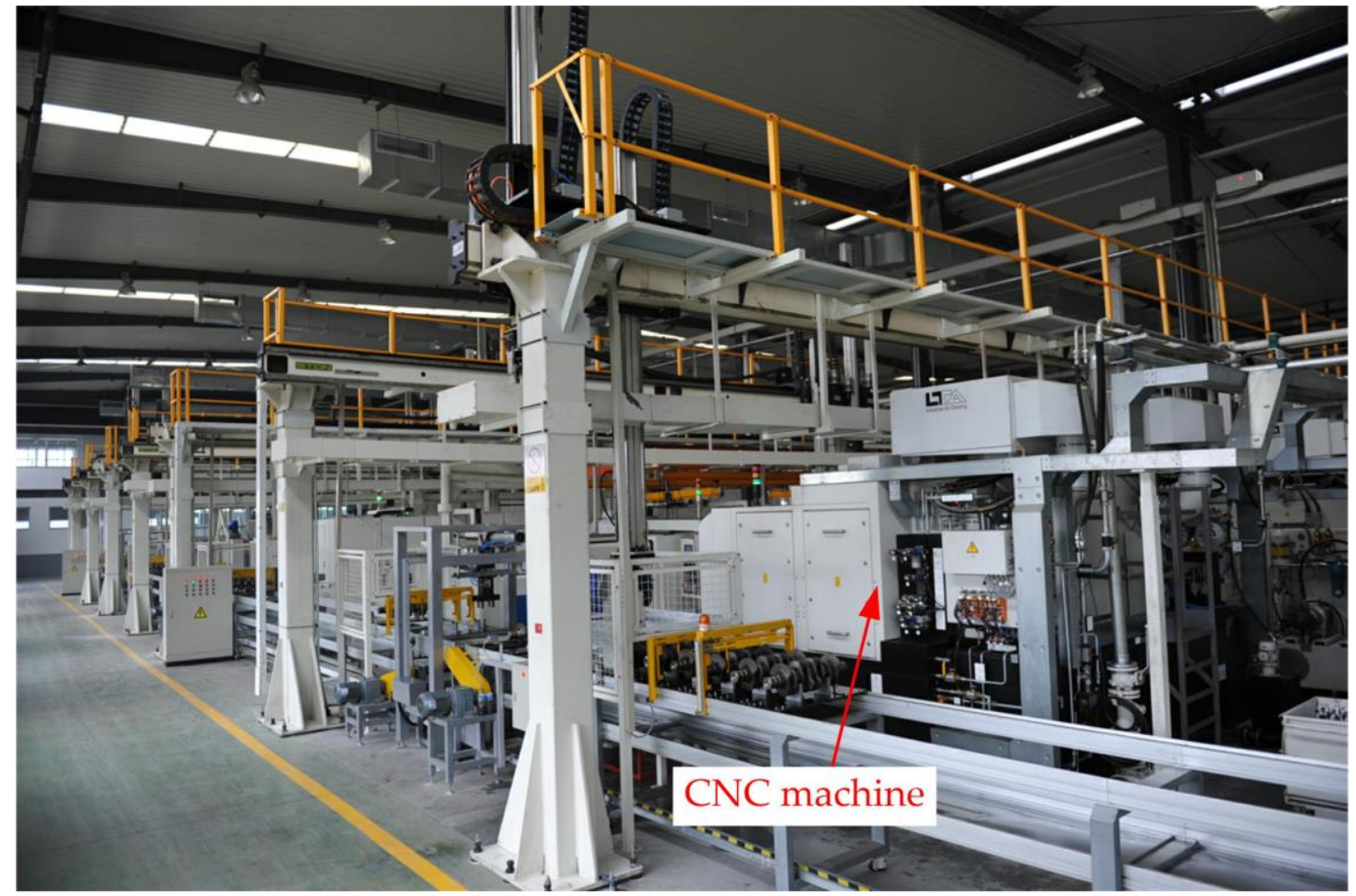

Figure 3. The scene of the automotive part production line. 
Table 1. The failure rate of each machine.

\begin{tabular}{cc}
\hline Machine & Failure Rate $\boldsymbol{\lambda} \mathbf{( 1 / h )}$ \\
\hline M01 & 0.0022 \\
M02 & 0.0017 \\
M03 & 0.0026 \\
M04 & 0.0026 \\
M05 & 0.0027 \\
M06 & 0.0021 \\
M07 & 0.0021 \\
M08 & 0.0025 \\
M09 & 0.0021 \\
M10 & 0.0034 \\
M11 & 0.0023 \\
M12 & 0.0020 \\
M13 & 0.0027 \\
M14 & 0.0026 \\
M15 & 0.0023 \\
M16 & 0.0035 \\
M17 & 0.0017 \\
M18 & 0.0019 \\
M19 & 0.0054 \\
M20 & 0.0030 \\
\hline
\end{tabular}

\subsection{Evaluating of Machine Criticality for the Automotive Part Production Line}

In this case study, the number of uniform design factor $S$ was equal to 20 (the number of machines in the automotive part production line) and the value of level $N(N$ is 3-5 times $S$ ) took 70 , so the uniform design table $U_{20}\left(20^{70}\right)$ was gained and used. It was assumed that the preventive maintenance had the same influence on the failure rate of each machine, namely, the failure rate of each machine uniformly reduced on the basis of current level as showed in Table 1. The 70 groups of factor levels for the machines' failure rate are listed in Table 2.

Table 2. The levels of the uniform design for the machines' failure rate.

\begin{tabular}{|c|c|c|c|c|c|c|c|c|}
\hline \multirow{2}{*}{ No. } & \multirow{2}{*}{ Machine } & \multicolumn{7}{|c|}{ Levels $\lambda_{j K}(1 / h)$} \\
\hline & & 1 & 2 & $\cdots$ & $j$ & $\cdots$ & 69 & 70 \\
\hline 1 & M01 & 0.0022 & 0.00218 & $\cdots$ & $0.0022-(j-1) \times 0.00002$ & $\cdots$ & 0.00084 & 0.00082 \\
\hline 2 & M02 & 0.0017 & 0.00168 & $\cdots$ & $0.0017-(j-1) \times 0.00002$ & $\cdots$ & 0.00034 & 0.00032 \\
\hline 3 & M03 & 0.0026 & 0.00258 & $\cdots$ & $0.0026-(j-1) \times 0.00002$ & $\cdots$ & 0.00124 & 0.00122 \\
\hline 4 & M04 & 0.0026 & 0.00258 & $\cdots$ & $0.0026-(j-1) \times 0.00002$ & $\ldots$ & 0.00124 & 0.00122 \\
\hline 5 & M05 & 0.0027 & 0.00268 & $\ldots$ & $0.0027-(j-1) \times 0.00002$ & $\ldots$ & 0.00134 & 0.00132 \\
\hline 6 & M06 & 0.0021 & 0.00208 & $\cdots$ & $0.0021-(j-1) \times 0.00002$ & $\cdots$ & 0.00074 & 0.00072 \\
\hline 7 & M07 & 0.0021 & 0.00208 & $\cdots$ & $0.0021-(j-1) \times 0.00002$ & $\cdots$ & 0.00074 & 0.00072 \\
\hline 8 & M08 & 0.0025 & 0.00248 & $\cdots$ & $0.0025-(j-1) \times 0.00002$ & $\cdots$ & 0.00114 & 0.00112 \\
\hline 9 & M09 & 0.0021 & 0.00208 & $\ldots$ & $0.0021-(j-1) \times 0.00002$ & $\ldots$ & 0.00074 & 0.00072 \\
\hline 10 & M10 & 0.0034 & 0.00338 & $\cdots$ & $0.0034-(j-1) \times 0.00002$ & $\cdots$ & 0.00204 & 0.00202 \\
\hline 11 & M11 & 0.0023 & 0.00228 & $\ldots$ & $0.0023-(j-1) \times 0.00002$ & $\ldots$ & 0.00094 & 0.00092 \\
\hline 12 & M12 & 0.0020 & 0.00198 & $\cdots$ & $0.0020-(j-1) \times 0.00002$ & $\cdots$ & 0.00064 & 0.00062 \\
\hline 13 & M13 & 0.0027 & 0.00268 & $\cdots$ & $0.0027-(j-1) \times 0.00002$ & $\cdots$ & 0.00134 & 0.00132 \\
\hline 14 & M14 & 0.0026 & 0.00258 & $\cdots$ & $0.0026-(j-1) \times 0.00002$ & $\ldots$ & 0.00124 & 0.00122 \\
\hline 15 & M15 & 0.0023 & 0.00228 & $\cdots$ & $0.0023-(j-1) \times 0.00002$ & $\ldots$ & 0.00094 & 0.00092 \\
\hline 16 & M16 & 0.0035 & 0.00348 & $\cdots$ & $0.0035-(j-1) \times 0.00002$ & $\cdots$ & 0.00214 & 0.00212 \\
\hline 17 & M17 & 0.0017 & 0.00168 & $\cdots$ & $0.0017-(j-1) \times 0.00002$ & $\cdots$ & 0.00034 & 0.00032 \\
\hline 18 & M18 & 0.0019 & 0.00188 & $\ldots$ & $0.0019-(j-1) \times 0.00002$ & $\cdots$ & 0.00054 & 0.00052 \\
\hline 19 & M19 & 0.0054 & 0.00538 & $\ldots$ & $0.0054-(j-1) \times 0.00002$ & $\ldots$ & 0.00404 & 0.00402 \\
\hline 20 & M20 & 0.0030 & 0.00298 & $\ldots$ & $0.0030-(j-1) \times 0.00002$ & $\ldots$ & 0.00164 & 0.00162 \\
\hline
\end{tabular}

The uniform design table was created using the DPS (Data Processing System) software (DPSv17.0, Hangzhou Ruifeng Information Technology Co., Ltd., Hangzhou, China), and 70 groups of combined 
schemes were gained, which were taken into the simulation model for the production line based on the Plant Simulation software (Plant Simulation 13.0, Siemens Ltd. China, Beijing, China). Then, the operating rate $Y$ of the production line with different groups of the machine failure rates was obtained. The regression equation between the failure rate of each machine and the operating rate of production line was established using MATLAB software (MATLAB 7.0, MathWorks Inc., Natick, MA, USA). The coefficients of regression equation are shown in Table 3.

Table 3. The coefficients of regression equation.

\begin{tabular}{ccc}
\hline Coefficient & Value & Corresponding Machine \\
\hline$a$ & 0.996 & Constant term \\
$b_{1}$ & -1.766 & M01 \\
$b_{2}$ & -1.897 & M02 \\
$b_{3}$ & -1.051 & M03 \\
$b_{4}$ & -1.129 & M04 \\
$b_{5}$ & -3.441 & M05 \\
$b_{6}$ & -1.231 & M06 \\
$b_{7}$ & -0.953 & M07 \\
$b_{8}$ & -1.551 & M08 \\
$b_{9}$ & -0.407 & M09 \\
$b_{10}$ & -1.060 & M10 \\
$b_{11}$ & -1.263 & M11 \\
$b_{12}$ & -1.848 & M12 \\
$b_{13}$ & -0.847 & M13 \\
$b_{14}$ & -0.914 & M14 \\
$b_{15}$ & -2.286 & M15 \\
$b_{16}$ & -1.405 & M16 \\
$b_{17}$ & -1.338 & M17 \\
$b_{18}$ & -1.437 & M18 \\
$b_{19}$ & -1.875 & M19 \\
$b_{20}$ & -1.977 & M20 \\
\hline
\end{tabular}

According to the coefficients of regression equation as shown in Table 3, it can be found that the coefficients of regression equation are all negative except for the constant term, that is, there is a negative correlation between the failure rate of each machine and the operating rate of the production line. The bigger the absolute value of the regression equation coefficient, the greater the influence of the machine failure rate on the operating rate of the production line. Therefore, the absolute value of the regression equation coefficient can be used as the criterion to evaluate criticality of the machines. On the basis of the ABC analysis method as mentioned in Section 2, four machines such as M02, M05, M15, and M20 were evaluated as the most critical machines and six machines such as M01, M08, M12, M16, M18, and M19 were evaluated as the secondary critical machines.

\subsection{Maintenance Period of Most Critical Machines for the Automotive Part Production Line}

The statistics and analysis were performed on the failure data and potential failure data of all most critical machines, and its results were taken into Equation (14); then, the delay-time function $F(h)$, the probability of potential failure $\theta$ and the probability of potential failure detected in preventive maintenance $r$ were gained, that is, $F\left(t_{h}\right)=1-\exp \left(-0.0098 t_{h}\right), \theta=0.8012$, and $r=0.4145$.

The integral transformation of Equation (9) was carried out and while $t=T_{n-1}, t+\Delta t=T_{n-1}+$ $T_{m}=T_{n}$. The expected number of the machine failure in the preventive maintenance period $T_{m}$ was expressed:

$$
E N_{f}\left(T_{m}\right)=\theta T_{m}-\frac{\theta \times r \times\left(e^{\eta T_{m}}-1\right)}{\eta \times\left(e^{\eta T_{m}}-1+r\right)}
$$


Equation (21) was taken into the decision objective Equation (4):

$$
E D\left(T_{m}\right)=\frac{t_{f} E N_{f}\left(T_{m}\right)+t_{p}}{T_{m}}=\frac{t_{f}\left[\theta T_{m}-\frac{\theta \times r \times\left(e^{\eta T_{m}}-1\right)}{\eta \times\left(e^{T^{T}}-1+r\right)}\right]+t_{p}}{T_{m}} .
$$

On the basis of the statistics and analysis of maintenance data, the average shutdown time of automatic production line caused by the most critical machines' failure was $2.4 \mathrm{~h}\left(t_{f}=2.4 \mathrm{~h}\right)$ and the average preventive maintenance time of the most critical machines was $1.5 \mathrm{~h}\left(t_{p}=1.5 \mathrm{~h}\right)$. Then, the parameters $F\left(t_{h}\right), \theta, r, t_{f}$ and $t_{p}$ were taken into Equation (22), and the relationship between the expected shutdown time of the production line and the preventive maintenance period was obtained by MATLAB software, as shown in Figure 4.

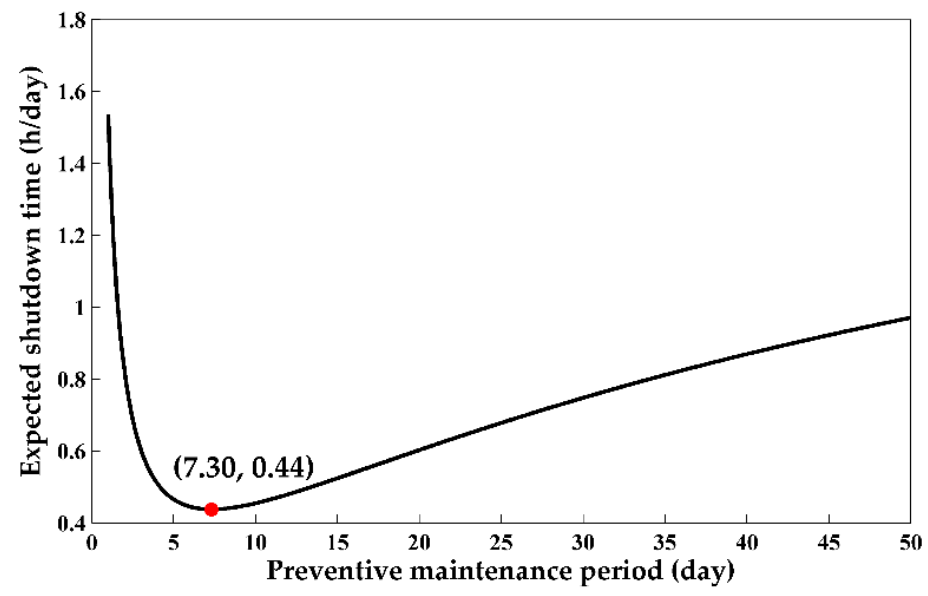

Figure 4. The relationship between expected shutdown time of the production line and the preventive maintenance period.

According to Figure 4, it can be found that the shutdown time is the shortest while the preventive maintenance period $T_{m}$ is 7.30 days and the value of shortest shutdown time is 0.44 . Considering of the actual operation of the production enterprise and simplifying the maintenance plan, the preventive maintenance period for the most critical machines (M02, M05, M15, M20) was designated as seven days.

In order to ensure the reliability and operating rate of the automatic production line, the reliability of each machine in the production line is not less than 0.7 , namely, the cumulative failure probability of each machine should be lower than 0.3 in this case study [25]. To the best of the authors' knowledge, there is no unified standard of minimum value of machine reliability now. Thus, in this case study, we referred to the study results of reference [25]. Thus, the Weibull distribution model of each machine and reliability of each machine were calculated when the $T_{m}$ was 7 . The results are shown in Table 4 .

Table 4. Reliability of the most critical machines during the preventive maintenance period.

\begin{tabular}{ccc}
\hline Machine & Weibull Distribution Model & Reliability (\%) \\
\hline M02 & $F_{w}(t)=1-\exp \left[-(t / 393.8)^{1.118}\right]$ & 0.783 \\
M05 & $F_{w}(t)=1-\exp \left[-(t / 442.6)^{1.410}\right]$ & 0.866 \\
M15 & $F_{w}(t)=1-\exp \left[-(t / 263.7)^{1.462}\right]$ & 0.751 \\
M20 & $F_{w}(t)=1-\exp \left[-(t / 454.5)^{1.784}\right]$ & 0.921 \\
\hline
\end{tabular}

It can be seen from Table 4 that the reliability of each machine in the preventive maintenance period are higher than 0.7. Therefore, while the preventive maintenance period of the most critical machine was designed as seven days, the production line had the shortest shutdown time and sufficient reliability. 


\subsection{Maintenance Period of Secondary Critical Machines for the Automotive Part Production Line}

As mentioned in Section 5.2, the cumulative failure probability of machine should be lower than the $F_{\max }$ and $F_{\max }=1-0.7=0.3$. The value of $F_{\max }$ was taken into Equation (16), and the preventive maintenance period $T_{S}$ under reliability constraints was gained, which was equal to 13 days. According to Equation (18), the cumulative failure probability of each secondary critical machine $F_{m}\left(T_{s}\right)$ during $T_{s}$ period were gained. The values of $F_{m}\left(T_{s}\right)$ for the secondary machine are shown in Table 5.

Table 5. The cumulative failure probability of secondary critical machines.

\begin{tabular}{cccc}
\hline Machine & $T_{\text {se }} /$ day & $T_{s} /$ day & $F_{m}\left(T_{s}\right) / \%$ \\
\hline M01 & 14.5 & 13 & 0.266 \\
M08 & 14.3 & 13 & 0.269 \\
M12 & 13.2 & 13 & 0.296 \\
M16 & 13.3 & 13 & 0.291 \\
M18 & 13.3 & 13 & 0.292 \\
M19 & 17.4 & 13 & 0.218 \\
\hline
\end{tabular}

As shown in Table 5, the cumulative failure probability $F_{m}\left(T_{s}\right)$ of each secondary critical machine is less than $F_{\max }\left(F_{\max }=0.3\right)$, while the preventive maintenance period $T_{s}$ is 13 days. According to Equation (20), the operating rate $Y_{m}$ is always equal to 1 with the values listed in Table 5. Hence, the preventive maintenance period of each secondary critical machine $T_{P M m}$ is 13 days $\left(T_{P M m}=T_{0} \cdot Y_{m}\right)$. Namely, six machines evaluated as the secondary critical machines (M01, M08, M12, M16, M18 and M19) were in a group for the preventive maintenance with the 13 days period.

The fixed cost $C_{0}$, the average cost of the parts replacement $C_{m}$, and the average cost of the breakdown maintenance $C_{f m}$ of each secondary critical machine in a preventive maintenance period were obtained from the maintenance data provide by the enterprise and shown in Table 6.

Table 6. The maintenance cost data of each secondary critical machine.

\begin{tabular}{cccc}
\hline Machine & $\boldsymbol{C}_{\mathbf{0}} \mathbf{( R M B )}$ & $\boldsymbol{C}_{\boldsymbol{m}} \mathbf{( R M B )}$ & $\boldsymbol{C}_{\boldsymbol{f}} \mathbf{( R M B )}$ \\
\hline M01 & & 275 & 3470 \\
M08 & & 370 & 2405 \\
M12 & \multirow{2}{*}{5100} & 300 & 3190 \\
M16 & & 330 & 2575 \\
M18 & & 315 & 2640 \\
M19 & & 290 & 3895 \\
\hline
\end{tabular}

When the preventive maintenance period $T_{\mathrm{S}}$ was 13 days and the production period was one year, there were about 26 periods for the preventive maintenance. The total maintenance costs could be calculated by Equation (19) and $T C\left(T_{S}\right)=353,306$ RMB. Compare with the results in literature [12,28], it could be found that, although the number of machines and the maintenance costs were different, the average maintenance cost of each machine in the present case study was lower. The preventive maintenance strategy for the secondary critical machine in this study should reduce the maintenance cost; meanwhile, the production line reliability was ensured.

\section{Conclusions}

(1) A maintenance strategy for automatic production line was developed on the basis of considering both the reliability, operating rate and maintenance cost. Furthermore, the different maintenance methods were employed according to the criticality of the machines in the production line. The maintenance strategy was practical. If this maintenance strategy was used to guide the maintenance and management work for the production enterprise, the economic efficiency and production efficiency of the automatic production line should increase obviously. 
(2) The uniform design method and simulation method were combined to establish the regression model between the failure rate of machine and the operating rate of the production line. The influence extent of the machine failure rate on the operating rate was used as the criterion to evaluate criticality of the machines. Then, the machines were classified into three groups: the most critical machines, the secondary critical machines and the general machines. The general machines were performed on the breakdown maintenance.

(3) The preventive maintenance model for the most critical machines was built based on delay-time theory. The relation between the preventive maintenance period for the most critical machines and total shutdown time of the production line was gained. The best preventive maintenance period of the most critical machines was determined by taking the shortest total shutdown time as the decision objective.

(4) The preventive maintenance model for the secondary critical machines was built based on the reliability-maintenance costs. The preventive maintenance period was gained according to take the parameters $\left(C_{0}, C_{m}, C_{f m}, F_{\max }, \alpha_{m}, \beta_{m}\right)$ into the model which were determined by the statistics and analysis of failure data and maintenance data of the secondary critical machines.

(5) A case study on an automotive part automatic production line was carried out. The results showed that the optimal preventative maintenance period for the most critical machines' group comprised of M02, M05, M15, and M20 was seven days while the production line reliability was ensured. In addition, the secondary critical machines' group comprised of M01, M08, M12, M16, M18, and M19 was 13 days while the average maintenance costs were lower.

(6) This study assumed that the system would be in a new state after the maintenance, but, in fact, this assumption was difficult to achieve under the limitation of maintenance capability. Therefore, it is necessary to investigate the synchronous imperfect preventive maintenance strategy for the automatic production line in the future.

Author Contributions: Supervision and Funding Acquisition, G.L.; Writing-Original Draft Preparation, Y.L. and X.Z.; Data Curation and Analysis, C.H. and B.X.; Writing-Review and Editing, J.H. and J.C.

Funding: This research was funded by National Science and Technology Major Project of the Ministry of Science and Technology of China (2017ZX04002001) and Jilin Scientific and Technological Development Program (20170204007GX, 20180312042ZG).

Acknowledgments: The work was supported by the program for the JLU Science and Technology Innovative Research Team (JLUSTIRT).

Conflicts of Interest: The authors declare no conflict of interest.

\section{Abbreviations}

$e$

$f(h)$

$m_{k l}$

$n$

$n_{k}$

$n_{m}$

$P_{k}$

$r$

$t_{\mathrm{f}}$

$t_{h}$

$t_{\mathrm{p}}$

$u$

$v(t)$

The number of the secondary critical machines

The delay-time function

The number of failures in the $l$-th interval of the $k$-th maintenance period

The number of preventive maintenance in the production period

The number of potential failures detected in the $k$-th maintenance

The number of preventive maintenance for the $m$-th secondary critical machine

The probability of the $n_{k}$-th potential failure detected in the $T_{k}$

The probability of potential failure which can be detected by preventive maintenance

The average failure shutdown time

The delay-time

The average preventive maintenance shutdown time

Time when a potential failure of machine generates

The failure occurrence rate at time $t$ 
The number of $\Delta t$ intervals

The fixed cost for each preventive maintenance

The average cost of the breakdown maintenance for the $m$-th secondary critical machine

The cost of parts replacement for the preventive maintenance of the $m$-th secondary critical machine The expected failure number for all most critical machines in the preventive maintenance period

The expected failure number of the $m$-th machine

The upper limit value of the cumulative failure probability

The cumulative failure probability of the $m$-th machine during the preventive maintenance period

A $\Delta t$ interval of the preventive maintenance period

The duration time of $k$-th maintenance

A production period

The preventive maintenance period of the most critical machines

The maximum preventive maintenance period of each secondary critical machine under reliability constraints

The preventive maintenance period of the $m$-th secondary critical machine

The preventive maintenance period of the secondary critical machines

The scale parameter in the Weibull distribution

The shape parameter in the Weibull distribution

The occurrence rate of potential failure

\section{References}

1. Kang, Y.-S.; Kim, H.; Lee, Y.-H. Implementation of an RFID-based sequencing-error-proofing system for automotive manufacturing logistics. Appl. Sci. 2018, 8, 109. [CrossRef]

2. Cristalli, C.; Grabowski, D. Multivariate analysis of transient state infrared images in production line quality control systems. Appl. Sci. 2018, 8, 250. [CrossRef]

3. Zhou, Z.; Dou, Y.; Sun, J.; Jiang, J.; Tan, Y. Sustainable production line evaluation based on evidential reasoning. Sustainability 2017, 9, 1811. [CrossRef]

4. Vuuren, M.V.; Adan, I.J.B.F.; Resing-Sassen, S.A.E. Stochastic modeling of manufacturing systems. Oper. Res. Spektrum 2005, 27, 315-338.

5. Doostparast, M.; Kolahan, F.; Doostparast, M. A reliability-based approach to optimize preventive maintenance scheduling for coherent systems. Reliab. Eng. Syst. Saf. 2014, 126, 98-106. [CrossRef]

6. Wang, W. An overview of the recent advances in delay-time-based maintenance modelling. Reliab. Eng. Syst. Saf. 2012, 106, 165-178. [CrossRef]

7. Shanmugam, R. Case studies in reliability and maintenance. J. Stat. Comput. Simul. 2003, 73, $276-277$. [CrossRef]

8. Wang, H.Z. A survey of maintenance policies of deteriorating systems. Eur. J. Oper. Res. 2007, 139, 469-489. [CrossRef]

9. Chen, Y.M.; Li, S.B.; Jin, B.B. Determination of cycle of mitering lock overhaul based on preventive group maintenance strategy. J. Southeast Univ. 2014, 44, 436-440.

10. Aghezzaf, E.H.; Najid, N.M. Integrated production planning and preventive maintenance in deteriorating production systems. Inf. Sci. 2008, 178, 3382-3392. [CrossRef]

11. Wu, F.; Niknam, S.A.; Kobza, J.E. A cost effective degradation-based maintenance strategy under imperfect repair. Reliab. Eng. Syst. Saf. 2015, 144, 234-243. [CrossRef]

12. Barata, J.; Soares, C.G.; Marseguerra, M.; Zio, E. Simulation modelling of repairable multi-component deteriorating systems for 'on condition' maintenance optimization. Reliab. Eng. Syst. Saf. 2002, 76, 255-264. [CrossRef]

13. Tong, J.; Mao, D.; Xue, D. A genetic algorithm solution for a nuclear power plant risk-cost maintenance model. Nucl. Eng. Des. 2004, 229, 81-89. 
14. Rao, A.N.; Bhadury, B. Opportunistic maintenance of multi-equipment system: A case study. Qual. Reliab. Eng. Int. 2015, 16, 487-500. [CrossRef]

15. Talukder, M.S.; Knapp, G.M. Equipment assignment to multiple overhaul blocks in series systems. J. Qual. Maint. Eng. 2002, 8, 319-330. [CrossRef]

16. Arunraj, N.S.; Maiti, J. Risk-based maintenance policy selection using AHP and goal programming. Saf. Sci. 2010, 48, 238-247. [CrossRef]

17. Zhao, F.; Wang, W.; Peng, R. Delay-time-based preventive maintenance modelling for a production plant: A case study in a steel mill. J. Oper. Res. Soc. 2015, 66, 2015-2024. [CrossRef]

18. Fang, K.T.; Lin, D.J.; Winker, P.; Zhang, Y. Uniform design: Theory and application. Technometrics 2000, 42, 237-248. [CrossRef]

19. Zheng, M.; Zhang, X.; Zhang, L.; Teng, H.; Hu, J.; Hu, M. Uniform test method optimum design for Drag-Type Modified Savonius VAWTs by CFD numerical simulation. Arab. J. Sci. Eng. 2018, 43, 4453-4461. [CrossRef]

20. Chen, R.Q.; Ma, S.H. Production and Operations Management; Higher Education Press: Beijing, China, 1999; pp. 302-304.

21. Moncadatorres, A.; Leuenberger, K.; Gonzenbach, R.; Luft, A.; Gassert, R. Activity classification based on inertial and barometric pressure sensors at different anatomical locations. Physiol. Meas. 2014, 35, 1245-1263. [CrossRef] [PubMed]

22. Teunter, R.H.; Babai, M.Z.; Syntetos, A.A. ABC classification: Service levels and inventory costs. Prod. Oper. Manag. 2010, 19, 343-352. [CrossRef]

23. Yuan, Y.; Fang, L.I.; Hou, C.Y.; Yang, L. Opportunistic group maintenance optimization of multi-unit system under dependence. Comput. Integr. Manuf. Syst. 2012, 18, 827-832.

24. Baker, R.D.; Wang, W. Developing and testing the delay-time model. J. Oper. Res. Soc. 1993, 44, 361-374. [CrossRef]

25. Das, K.; Lashkari, K.S.; Sengupta, S. Machine reliability and preventive maintenance planning for cellular manufacturing system. Eur. J. Oper. Res. 2007, 183, 162-180. [CrossRef]

26. Kardon, B.; Fredendall, L.D. Incorporating overall probability of system failure into a preventive maintenance model for a serial system. J. Qual. Maint. Eng. 2002, 8, 331-345. [CrossRef]

27. Nahas, N.; Nourelfath, M. Buffer allocation, machine selection and preventive maintenance optimization in unreliable production lines. In Proceedings of the 2015 IEEE International Conference on Industrial Engineering and Systems Management, Seville, Spain, 21-23 October 2015; Volume 28, pp. 1-9.

28. Afefy, I.H. Reliability-centered maintenance methodology and application: A case study. Engineering 2010, 2, 863-873. [CrossRef]

(C) 2018 by the authors. Licensee MDPI, Basel, Switzerland. This article is an open access article distributed under the terms and conditions of the Creative Commons Attribution (CC BY) license (http:/ / creativecommons.org/licenses/by/4.0/). 